\title{
The Transformative Role of Telemedicine on Coordination: A Practice Approach
}

\author{
Julien Meyer \\ Ryerson University \\ julien.meyer@ryerson.ca
}

\author{
Guy Paré \\ HEC Montréal \\ guy.pare@hec.ca
}

\begin{abstract}
Delivering coordinated care at a distance challenges work practices and interprofessional collaboration. Using a case study methodology, we analyzed how three occupational groups, pathologists, technologists, and surgeons, coordinate work during the deployment of a major telepathology network in Eastern Canada. The aim of this study is to determine the extent to which and how telemedicine modifies coordination practices.

Transformations emerged from our in-depth case analysis around three aspects of coordination: predictability, common understanding and accountability. First, predictability relied on routines in traditional settings, but shifted to a reliance on plans and rules in a telemedicine setting. Second, common understanding of the task shifted from relying on familiarity between stakeholders to an emphasis on standards. Third, accountability became less collective and more individual and contractual in a telemedicine setting, resulting in more marked boundaries between professional groups. Finally, proximity remained a determinant of accountability in telemedicine contexts, regardless of organizational arrangements. Implications for research and practice are discussed.
\end{abstract}

\section{Introduction}

Telemedicine, the use of telecommunications to diagnose and treat diseases and ill-health, has become a multibillion dollar business. Worldwide revenue is poised to grow from $\$ 19.2$ billion in 2014 to $\$ 43.4$ billion in 2019 [4]. One of the reasons for this growth is the potential of telemedicine to address some of the key challenges facing healthcare systems in developed countries, such as controlling the spiraling costs of care and extending accessibility of care. To harness potential benefits, healthcare organizations and care providers need to understand and adapt to this new way of delivering care services [20].
Telemedicine is more than a faster way to access existing healthcare resources; it also represents an organizational and social innovation [7]. Telemedicine can be conceived as a collaboration platform connecting experts to non-experts [5]. For it to succeed, health care providers need to integrate telemedicine activities to health care service delivery [16] and to remotely coordinate [16,22]. Coordination is essential to the effective delivery of care. The quality of care coordination is associated with patient outcomes and the overall performance of health systems. Achieving better coordination has drawn growing interest from researchers and health care accreditation bodies [31]. In short, coordinating the provision of care services is essential when telemedicine is introduced in health care settings.

A practice approach that focuses on activities and actions rather than on formal decision making structures is appropriate in environments where there is uncertainty, complexity such as in health care delivery [11]. It draws attention to the human interactions during coordination. In this approach, coordination can be defined as "a temporally unfolding and contextualized process of input regulation and interaction articulation to realize a collective performance" [21:1157].

In a seminal article, Barley shows how the introduction of a new IT-based artefact (a CT scanner) triggered a transformation in the interactions between technicians and radiologists, and a change in institutional roles and responsibilities [1]. His findings suggested that unintended consequences can lead to new patterns of action (what he calls scripts) that subsequently reify into structural transformations, such as in roles and status.

Telemedicine, by enabling coordination across organizational boundaries, initiates such a structuring process. Medical work remains largely organized around the premise of colocation, and this premise has cultural, legal and practical ramifications [21]. Telemedicine challenges two cornerstones of medical practices: the patient-clinician encounter and the assumption that a care episode takes place within a 
single geographical setting (e.g., the hospital) [3]. Accountability, the assignment of responsibility for each elements of the task to collectively achieve, is crucial for coordinating work [23]. Telemedicine often requires the transfer of some medical tasks from doctors to other personnel. For instance, in a home-telemonitoring program for cardiac patients, nurses were accountable for transferring information from patients monitored at a distance to physicians. However, their role as an intermediary also extended to interpreting clinical information [20]. For instance, nurses ignored impossible vital signs mistakenly recorded and transmitted by patients. As the home telemonitoring system essentially replaced some doctor-patient encounters, nurses had to make concerted efforts to account for their work and the doctors' prescribed treatment, thus stretching to its limits the principle that "a doctor is always in charge". Telemedicine may also alter the meaning and purposes of professional roles. In a telecardiology project, the initial goal was to provide patient access to cardiology specialists in order to prevent and deal with cardiac emergencies. Instead of achieving this goal, the telecardiology project reoriented to a social practice of reassuring patients about their condition and reassuring general practitioners about their decisions [12].

In sum, prior research provides us with some insights into how telemedicine transforms the fabric of work practices in healthcare organizations, but there still lacks a clear account of the transformations as they relate to coordination. The objective of the present study is to develop a theory of how telemedicine transforms coordination. We focused on stakeholders' actions and on the context of coordination. To achieve our main research objective, we analyzed the changes in coordination practices as perceived by three occupational groups, pathologists, technologists, and surgeons, during Intraoperative Consultations (IOCs). Our empirical investigation attempts to answer the following research question: to what extent and how does telemedicine alter coordination practices in the context of IOCs?

The remaining of this paper is structured as follows. In the next section, we present the research methods and setting. This is followed by a presentation of the coordination practices used in traditional and telepathology settings. We then analyse the transformations in three major aspects of coordination, namely, predictability, common understanding, and accountability. We conclude by discussing the main contributions of our work to both research and practice, and its methodological limitations.

\section{Methodology}

We followed an inductive approach based on a single case that is particularly revealing and critical $[24,30]$. The focus is on the everyday practices used to coordinate pathology-related work before and after the introduction of telemedicine. Our respondents are the clinicians involved in telepathology episodes, namely, pathologists, surgeons, and technologists.

\subsection{Research setting}

The particular form of telemedicine that we investigated in this study is telepathology which can be simply defined as the practice of pathology at a distance. Pathology is the branch of medicine that study the nature of diseases and its causes. Pathologists examine slides of human tissue to uncover the presence of disease, notably cancers [28]. They work in close collaboration with other clinicians. Surgeons extract tissue specimens to be diagnosed, and laboratory technologists take these specimens to prepare the glass slides that are examined by pathologists under a microscope to diagnose diseases. As explained in detail later, pathologists, surgeons and technologists sequentially perform interdependent tasks, which sometimes require fast and unexpected responses.

With telepathology, instead of being examined through a microscope, the slide is scanned, pathologists view the virtual slide using a highresolution computer screen and then they perform a diagnosis from the image rather than from the physical artifact [29]. More details of the practical aspects of the telepathology environment are provided in the results section.

The telepathology project under study is that of a regional healthcare network located in Canada, for a territory the size of Germany, but inhabited by only about 2 million people unequally spread between a dense urban center with a university hospital and barely populated remote regions served by small regional hospitals. A total of 48 pathologists and 17 sites are targeted by the project, which makes it one of the largest telepathology networks in the world. Moreover, although 33 of the pathologists are located at the university hospital, the project has no central site responsible for providing pathology expertise throughout the network. In other words, each site is responsible for developing and negotiating Service Level Agreements (SLAs), i.e. formal contracts with other sites committing to provide or receive pathology services using telepathology. Such decentralized telepathology networks are associated with heightened coordination challenges [18]. The 
technology was gradually deployed across the network in late 2010 and early 2011.

\subsection{Data collection and analysis}

Our data collection involved a total of 12 site visits between 2012 and 2015. We relied on interviews and observations to strengthen the grounding of theory through triangulation [30]. A total of 60 one-to-one interviews were conducted by three interviewers with 51 different respondents stemming from 14 different hospitals (some over the phone), leading to over 44 hours of recording. We mainly relied on semi-structured interviews with key informants, the goal being to understand coordination in traditional, collocated settings and in a telepathology context. The sampling strategy was a mix of snowball and maximum variation [24,26]. Semi-structured interviews, were conducted until we reached theoretical saturation [10], with a focus on coordination practices when working in traditional, collocated settings and when using the telepathology system. We remained open to emerging insights and concepts that would help explain transformations. We also spent several days in hospital laboratories, enabling us to directly observe stakeholders coordinating in situ, and to access documents and other material involved in these interactions.

We followed the principles of interpretive case study research [24]. Our goal was to inductively build an explanation of the transformations in coordination practices. The first step was to write field notes and to develop an in-depth understanding of how coordinating was achieved. The interviews were transcribed verbatim and we initially oriented our analysis towards describing the case, in terms of coordinating in traditional and in telepathology settings [30]. One of the interviewer then developed a coding scheme, using Nvivo, to capture the transformations in coordination. Coding was oriented towards action and the verb form to highlight the practice perspective and the standpoint of the stakeholder.

In the following section we provide a detailed description of the case study. More specifically, we present how telepathology has materially transformed the work environment. We then explain and illustrate the IOC practice in a traditional and in a telepathology setting.

\section{Case description}

\subsection{The material transformations in the work environment}

Telepathology introduces substantial material changes to the pathology laboratory environment. These changes are both mechanical and digital [2]. The mechanical changes involve the introduction of new equipment: in the laboratory, a macroscopy workstation, a scanner, a computer station, and, in the pathologist's office a high definition screen dedicated to the video conference. During the system installation stage, a dedicated space is cleared to accommodate the new devices. The macroscopy workstation allows the technologists to manipulate the patient specimen under the supervision of the distant pathologists using the communication system. Local laboratory technologists are responsible for keeping operational the telepathology material, along with the rest of the laboratory equipment. It is typically installed in the laboratory itself, an environment deemed "contaminated" by its contact with biological specimens.

For its part, the digital materiality consists of the telepathology software applications, used to scan and consult the slides, as well as communicating about the process. These are proprietary interfaces designed by the system providers. The laboratory environment revolves around the manipulation of physical artifacts: specimens, tools to manipulate them, and chemical components to alter their properties. In contrast, the computer and the scanner are used can be installed inside the laboratory or in any room nearby.

In traditional settings, the pathologist's office is typically across the hall from the laboratory where technologists work, and verbal exchanges are frequent. The pathologist office is uncontaminated and dominated by two contrasting devices: the computer and the microscope. Traditionally, the microscope is used to interpret the slides, while the computer is used for the pathologist's other tasks, such as email or updating electronic records (or physical ones through printing). With the digitization of physical slides, pathologists interpret digital images from a computer interface. In parallel, they keep using the microscope, which remains the preferred medium for local, routine slides as it is considered faster and more convenient [19].

The slide in itself is produced by physical manipulations in the laboratory, but it is "fixed": it has lost its "contaminating" property. The digitization of slides is another step towards rendering the slide easier to manipulate, transfer and conserve. A digital slide can be accessed immediately from any authorized screen in the network, by multiple people at the same time, and it doesn't degrade over time. The downside is the loss of potential biological manipulations on the physical slide, such as the 
possibility to modify colorations. Therefore, pathologists need the original specimen for some cases in order to provide a reliable diagnosis.

In short, the telepathology system offers new affordances in accessing and sharing information. But it also becomes the forced communication channel for pathologists' interventions.

\section{Case findings}

In this section, we introduce how stakeholders coordinate IOC at each step of the process. We first present coordination practices in traditional settings, followed by telepathology settings. Table 1 summarizes the coordination practices.

\subsection{Coordination in traditional settings}

In an IOC, the whole process of extracting, preparing and diagnosing a specimen is performed during the timeframe of a surgery. Typically, the goal of an IOC is to inform the surgeons whether a cancerous tumor has been fully removed and the surgery can be terminated, or if not, how they should pursue it $[25,27]$. In the absence of an on-site pathologist, the surgeon has three options: 1assuming the worst and performing a more aggressive surgery, 2- transferring the patient for surgery in a hospital with a pathologist, or 3operating in two steps, sending the specimen by courier to a remote pathologist and then performing a second surgery if necessary. IOC are generally planned ahead of time but may also be unplanned, when unexpected developments during a surgery require a pathology consultation. It is a highly collaborative process going from planning the surgery and extracting the specimen in the operating room, to providing a diagnosis to the surgeon under stringent time constraints.

The first step in a planned IOC is to plan it. Planning ensures the availability and readiness of laboratory resources during the surgery. In traditional settings, planning is generally performed in the lab by interpreting the operating room schedules, as pathology requests can generally be deduced from the schedule. For instance, if it mentions a lobectomy, the surgeon will likely require an IOC. Surgeons fill surgery requests in order to book operating rooms. The schedule is then accessed and interpreted by technologists, pathologists or lab secretaries in some cases, who infer IOC cases and plan further action without confirmation from the surgery team.

"Basically, the day before, we receive the operating procedure. It is the list of all the surgeries planned for the day after, with the name of all patients, and the surgeons who are going to perform those surgeries. According to the type of surgeries that are going to be performed, we are able to guess which ones will require IOC." A pathologist.

Surgeons may phone the lab to warn them, but overall, they don't need to formally request pathology support. In simple cases, technologists may not even inform the pathologists, who receive the slides in their office, ready to be read, without prior notice. The day of the surgery, an operating room nurse signals the start of the process by warning the lab that the surgery has started (C.). Typically, they would phone the technologists, who in turn phone the pathologists. This is done well before the surgeon extracts a specimen from the patient and has it transferred to the lab.

Upon reception, small specimens (e.g., brain specimens) are directly sliced into slides ready to be handed to the pathologist. Small specimens can be handled by technologists without any supervision. On the other hand, what are called large specimens (e.g., an intestine section) need to be handled, oriented and colored, to produce blocks of tissue in a process called macroscopy. For the more complex of these specimens, such as full breasts or intestines, macroscopy requires medical expertise. Macroscopy is a task shared between technologists handling the simple cases, and pathologists handling the complex ones. The technologist receiving the specimen in the lab determines whether an expert intervention (D.) by a pathologist is required for the macroscopy task. If that is so, the technologist stages the specimen on a macroscopy workstation, and calls the pathologist, who comes to the lab, manipulates, orients and describes the specimen, sometimes clarifying doubts with the surgeon over the phone.

Once a glass slide is prepared, it is ready to be examined by the pathologist through a microscope. IOC slides are part of the continuous daily load of routine slides coming from the lab to the pathologist office. Pathologists sometimes remain in the lab, in which case the technologist directly hands the slides (F.). But generally, the pathologists are in their office, and physical slides (IOC and routine cases) are carried to the pathologist by the technologist. To make this transfer clearer, less time-consuming and less disruptive, technologists organize batches of slides, for multiple patients, into folders. Technologists and pathologists have organized over time those folders in order to facilitate their work. All the slides from a patient are gathered on the same page, and within a page, slides are ordered in the logical order in which they should be viewed, such as the area they were extracted from. Color stickers on the folder edge denote the nature of the cases or their 
urgency. Technologists hand the folders over to pathologists following a routine schedule. IOC (as other urgent cases) are generally brought immediately (G.), but still organized in folders following the same principles.

"We used to carry the (IOC slides) to (the pathologist) office. He was at his desk doing microscopy and other routine daily cases. We brought the slides and told him: "This is your IOC". So he dropped his business and started immediately (working on the IOC) and communicated directly with the operating room to provide the results" A technician.

Pathologists then examine the slides, produce a diagnosis and finally call the surgeon waiting in the operating room (I.). Once the diagnosis is communicated, the surgeon may request additional exams, or terminate the IOC.

\subsection{Telepathology-based coordination}

Telepathology is the only way to get a diagnosis from a distant pathologist within the timeframe of a surgery. This makes IOC a key motivation for adopting and using telepathology. Between January 2011 and March 2015, 1,843 slides were scanned for IOC purposes over the telepathology network. Telepathology-based IOC involve a surgery team, a technologist in the local laboratory, and a distant pathologist.
At the planning stage, surgeons must actively request an IOC. They have to make a formal IOC service request (B.) at least one day in advance.

"We ask (the surgeons), as part of our clinical protocols, to indicate the cases for which they believe they will need an IOC. The on-duty pathologist receives the list of IOC cases the day before to be able to plan his work." A pathologist.

As explained earlier, the telepathology system comprises a dedicated videoconference system to support technologists while performing macroscopy. The pathologist sees the specimen, talks to the technician handling it, and can even draw indications over a specimen image. Still, the system has its constraints: a local technologist needs to physically handle the specimen and the pathologists are unable to palpate the specimen, which is sometimes necessary to identify a cancerous node. As one pathologist puts it, "technologists become our hands".

The physical absence of the pathologist creates an ambiguity as to who should physically manipulate complex cases. Technologists are expected to perform macroscopy, with surgeon supervision for complex macroscopy cases. But technologists are already present in the lab, are more available than surgeons busy in the operating room, have experience with macroscopy in general, and are familiar with the pathologists. This leads them to take an extended role in macroscopy, and to perform most complex macroscopy under distant pathologist supervision.

Table 1. Traditional versus telepathology-based coordination practices during an IOC

\begin{tabular}{|c|c|c|}
\hline Step /Setting & IOC in traditional setting & Telepathology-based IOC \\
\hline $\begin{array}{l}\text { 1. Planning } \\
\text { IOC }\end{array}$ & $\begin{array}{l}\text { A. Schedules interpretation. } \\
\text { Technologists and pathologists } \\
\text { anticipate and plan IOC from operating } \\
\text { room schedules }\end{array}$ & $\begin{array}{l}\text { B. Formal request. Surgeons are responsible for } \\
\text { requesting impending IOC from the lab at least a } \\
\text { day before the surgery }\end{array}$ \\
\hline \multirow[t]{2}{*}{$\begin{array}{l}2 . \\
\text { Macroscopy }\end{array}$} & \multicolumn{2}{|c|}{$\begin{array}{l}\text { C. Direct information by phone. Technologists are informed of IOC by surgeon/nurses. } \\
\text { Technologists phone the pathologists when they get the sample. }\end{array}$} \\
\hline & $\begin{array}{l}\text { D. Expert intervention. Pathologists } \\
\text { come to the lab to perform macroscopy } \\
\text { on complex cases }\end{array}$ & $\begin{array}{l}\text { E. Expert supervision. Technologists or surgeons } \\
\text { handle complex cases using the macroscopy station } \\
\text { under distance supervision from the pathologist }\end{array}$ \\
\hline $\begin{array}{l}\text { 3. Slide } \\
\text { preparation }\end{array}$ & $\begin{array}{l}\text { F.Collocated Handover } \\
\text { G. Handover through a directed folder. } \\
\text { The technologist hands personally over } \\
\text { the slides, organized in a folder }\end{array}$ & $\begin{array}{l}\text { H. Handover through System Upload } \\
\text { The telepathology system notifies the pathologist of } \\
\text { an uploaded slide }\end{array}$ \\
\hline \multirow[t]{2}{*}{ 4. Diagnosis } & $\begin{array}{l}\text { Pathologists diagnose the slide using } \\
\text { their microscope }\end{array}$ & $\begin{array}{l}\text { Pathologists diagnose the slide via their computer } \\
\text { screen }\end{array}$ \\
\hline & \multicolumn{2}{|c|}{ I. Expert conclusion sharing. The pathologist phones the diagnosis to the surgeon. } \\
\hline
\end{tabular}

After the glass slides are prepared, technologists go to the scanner room, log into the system, load the slides into the scanner, scan them, check the image quality, upload the digital slides to the pathologists and inform them. Then, the pathologists must log into the telepathology system, select the case and the 
slides before consulting them. The system then notifies the pathologist that digital slides are available. The case priority is managed either by the technologist tagging a slide "urgent" in the system, or by phoning the pathologists to inform them of the online availability of the slides. Alternatively, the pathologist may keep an open phone line with the lab, simply muting their own microphone after macroscopy is over, in order to be aware of when the slide is ready.

The telepathology system automatically attaches the metadata (the information about the slide, such as patient name, time of collection, slide nature or priority) to the digital slide. Pathologists click on an incoming case and view the digital slides displayed in the order dictated by the application, leading to an extra task of sorting them for viewing after the diagnosis.

"When I receive glass slides, they are already sorted. The slides go from A to A1, B1 to B20, etc. and I look at them in order, I have the sequence of the examined case. When I receive (complex cases) by telepathology, often, they are not in order and I can't rearrange the pictures." A pathologist.

The pathologists then phone the surgeons waiting in the operating room to inform them of the diagnosis. A direct verbal exchange with the surgeon is critical. Not even a nurse present in the operating room can pick up the phone to relay the diagnosis. It has to be the surgeon (or exceptionally a resident).

\section{Extent and nature of changes in coordination practices}

In this section, we analyze the major transformations in coordination that occurred during the shift from traditional to telepathology-based settings. How stakeholders coordinate in traditional and telemedicine settings was analyzed inductively, leading us to formulate a preliminary set of research propositions around three notions essential to coordination: the transformations in predictability, in common understanding and in the accountability of coordination practices. Each of these notions will be examined in turn.

\subsection{Predicting coordination through plans rather than routines}

Pathology labs, faced with huge amounts of repetitive tasks, rely heavily on routines to coordinate. In traditional settings, anticipating the workflow is essential in an environment where a large number of specimens need to be dealt with, even in the smallest labs. The bulk of activity in labs is repetitive and labs rely on highly structured practices to make these workflows predictable and efficient. Laboratory practices require frequent interactions between pathologists and technologists, rarely formalized, but instead the product of negotiated and proven routines, such as technologists referring macroscopy to pathologists (D.), taking the slide folders to the pathologist office (F.), or handing slides directly to the pathologist within the laboratory (F.). Routines also shape objects. The format of the glass slides, their coloration, slicing and arrangements in folders, are standardized and organized to facilitate interpretation by pathologists. The daily and steady repetition of requests enables technologists and pathologists to interpret incoming IOC requests from the local operating room schedules, but not from distant hospitals.

"In (our hospital), we know what the surgeries the following day will be. We are used to them, we know what types of surgeries will be done for what diseases, and that for that type of surgeries, the surgeon will want to ask this or that question. And to have that answer, an IOC needs to be done. By habit. (...With the distant hospital), I am not able to say "there may be a need for an IOC." I don't have the operating room schedule, and maybe, too, there won't be a need either." A pathologist.

At a distance, the increased variance in slides, people and processes challenges the predictability of lab work. Setting up routines for infrequent and complex tasks is difficult, and telepathology is by design used for rare cases: otherwise, the hospital would hire a local pathologist. This instability hinders the possibility to set up reliable routines. Telemedicine poses a challenge to the predictability of work. "People are afraid because they can't escape accountability. They need to be sure of the process and of the quality of the work done on the other side. Because it is a black box for us" A pathologist.

One of the major adaptations to the unpredictability of the environment in a telemedicine setting, and to the difficulty of setting up routines, is to rely more on formal plans and rules.

"We write down the process from start to end. We map it. (...) Then, pathologists validate the whole process, the quality, the information to share and the timelines. Everything needs to be listed and written." A pathologist.

From this shift away from routines to more formal plans and rules, we deduce a transformation in how predictability is achieved in order to coordinate.

Proposition 1: The introduction of telemedicine leads to a shift from routines to plans and rules, in 
order to create the predictability necessary to coordinate effectively.

\subsection{Coordinating with unfamiliar stakeholders}

Telemedicine also transforms how the coordinating stakeholders understand each other. During an IOC in collocated settings, proximity between pathologists and technicians facilitates coordination. Seeing others working helps understand and monitor each other in hospital settings [15]. Copresence is a straightforward way to achieve visibility, as in the handovers within the laboratory (F.), where seeing the pathologist around substitutes for the need for any other coordination mechanism. At first glance, visibility seems the first casualty of telemedicine coordination. But visibility goes beyond physical collocation. In both traditional and telepathology settings, clinicians resort to phones to communicate important information, such as communicating the start of the surgery and the diagnosis itself (C., I.). Using the phone, a synchronous rich communication device [8], creates visibility, reassures that proper action will be taken, and that accountability for the following step has been transferred. The adoption of telemedicine did not significantly alter those practices, even though alternatives such as emails are available. In that sense, proximity in terms of visibility can be replicated or even improved at a distance, by technological means.

But telemedicine reduces proximity in a much more critical way, that is, by reducing familiarity between the parties involved. Next to visibility, familiarity is another form of proximity enabling coordination [23]. Familiarity encompasses the relational aspect of coordination [13]. It allows people in the lab to interpret schedules in traditional settings (A), and to hand over slides within the lab (G). It helps determine which pathologists are covering IOC for which sites: "We (pathologists) said: 'if you have problems, we can do the IOCs'. It's better if we take care of them. It is always better to develop a proximity link. People know each other, are used to working together" A pathologist.

At a distance, familiarity is low. The informality, knowledge and trust created by working in close proximity are not easily replicated by technological means, and technologists have expressed fears of having to work with pathologists they barely know and with whom they have limited common understanding.

To ensure a common understanding despite the lack of familiarity, pathologists put an emphasis on inter-institutional standards and protocols compared to traditional laboratories.

"For a long-term Service Level Agreement, we need to organize, to have standardized processes. Knowing what phone number to call in case of problems, like the scanner doesn't work (...). When there are few stakeholders, one-to-one, it is easier. But when there are 10 people on the other side and we are 20 here, it is worth writing things and reaching a clear agreement" A pathologist.

Or, for example, pathologists in two different hospitals will exchange different types of colorations and decide together which ones to apply for both sites. This standardizes processes for technologists, for whom standardization means a consensus between pathologists at different sites.

Proposition 2: The introduction of telemedicine leads to a shift from familiarity to standards to create the common understanding necessary to coordinate effectively.

\subsection{Shifting forms of accountability within and between professions}

Telemedicine also subverts the role of proximity in medical practices and reframes the way in which activities are made accountable [21]. Accountability enables coordination by clarifying who is responsible for what aspect of the collective performance, and the nature of the relations between stakeholders [23].

Traditionally, pathologists are accountable for cases originating from their own institution. The spirit of telepathology is to abolish distance as the principle of accountability. Cases can in theory be dealt according to their priority and the pathologists' skills, rather than their location. "We are going to set up priorities for a set of laboratories. For instance, we are going to say: the first thing to do are urgent biopsies. Currently, this is not what is going on" (a pathologist). Doing so directly conflicts with the traditional principle of local accountability, expressed by some other pathologists. For them, accountability clearly remains local, and they assess the value of telepathology by the extent to which the technology helps them address local needs better. "When we learned that to get the technology, we would have to support another center, we were much less excited. We thought: "We are interested in getting helped, but we have nothing to gain at helping "', (a pathologist).

Referring and consulting hospitals involved in the telepathology project signed Service Level Agreements to ensure distant accountability for IOCs. Despite such formal contracts, pathologists did not always display the same accountability towards distant cases as towards local ones. In one particular 
instance, a surgeon experienced excessive delays in getting IOC, up to two hours, with a patient in the operating room. Pathologists in the consulting center were giving the distant IOCs a lower priority than less urgent local cases, leading to delays. Patients from local hospitals may have a higher priority than those served by telepathology [7]. The presence of objects and digital mediation can reduce the need for face-to-face engagement, leading to distance and neglect [2]. The problem was solved by assigning a dedicated pathologist to address IOCs from that hospital.

Service Level Agreements take place in a context of a geographical perception of accountability. In another instance, after a consulting center met recruitment issues, its pathologists stopped helping two remote hospitals until those issues were addressed. And some overloaded pathologists protected themselves from new responsibilities by opposing telepathology adoption in their hospital. They feared to inherit the responsibility to address requests from remote locations. This suggests that the geographical barriers removed by digitization play a regulating role.

Proposition 3: Stakeholders display more accountability for collocated than for distant coordination, regardless of organizational arrangements.

Proximity also plays a key role in defining the extent of accountability and in enabling overlaps of accountability in work practices. In traditional settings, technologists and pathologists take the responsibility for anticipating IOCs. They are familiar enough with local practices to interpret operating room schedules, feel accountable for local pathology activity, and therefore take the initiative of anticipating needs (A.). Proximity enables implicit coordination, anticipating the need or dynamically adjusting to the needs of other stakeholders without concertation between them [6]. This can play a decisive role in collective performance [17].

Telepathology-based coordination undermines this collective and implicit dynamic. Between pathologists and surgeons, distance pathologists are less comfortable with interpreting operating room schedules, and surgeons, rather than the laboratory team, became accountable for specifically requesting their IOC needs.

Pathologists can feel uncomfortable about their lack of control of what happens in the distant labs, and don't feel accountable for them.

"We have our own laboratory, we see what is produced there and if certain things are not correct, we try to settle the problem. These are our problems.
But with distant laboratories, this is not my problem. I do business with them during telepathology, but for the rest, I have nothing to say." A consulting pathologist.

Telemedicine redistributes this accountability towards local actors [25]. "Telepathology led technologists like us to perform tasks normally dedicated to pathologists" (a technologist). By performing the macroscopy, technologists may put themselves at legal risk, although no instance of technologists running into problems was reported. In short, telepathology undermines the "circulating accountability" held collectively in a ward (for local care) [20]. Proximity partially blurs the boundaries in accountability between professions, while telemedicine highlights them. Who manages the lab, who initiates requests, who is accountable for a macroscopy manipulation, need clear owners.

Proposition 4: The introduction of telemedicine sharpens the boundaries between professional groups, leading to an accountability that is less collective and more individual and contractual.

\section{Discussion}

Predictability, accountability, and common understanding have been identified in prior research as key constructs to analyze coordination [23]. In their view, these are three integrating conditions for coordination. Different coordination mechanisms, based on routines, plans, roles, proximity or objects, aim at achieving one or several of these conditions. Fulfilling these conditions makes coordination possible. These constructs proved relevant to study the impact of telemedicine on coordination. Predictability, common understanding and accountability emerged as axis along which the transformations unfolded, Predictability that used to rely mostly on routines is achieved through plans and rules in a telemedicine setting. As distant stakeholders are less familiar with each other, they rely on standards in order to restore common understanding. Finally, accountability is transformed, as it becomes more individual, contractual or rolebased, and less collective, while proximity remains a determinant of accountability even in telemedicine settings. While our findings confirm the conceptualization of coordination mechanisms (routines, plans, proximity, objects, roles) as substitutable mechanisms, the three integrating conditions for coordination are better defined as dimensions of coordination instead of competing or complementary conditions. Coordinating always involves some level of predictability, common 
understanding and accountability. As sensitizing devices, they draw the researcher's attention towards diverse aspects of coordination.

Our study also furthers our understanding of the link between technology and boundary-spanning coordination. Prior research suggests that technology can be an occasion for reconfiguring those boundaries [1], leading to cooperation, neglect or strain [2]. We found that technology can also be an opportunity for revealing boundaries, such as the macroscopy acts that pathologists, unlike technologists, are able to perform, but that technologists were performing anyway under pathologist supervision.

Our findings also bring valuable insights to practitioners and organizations switching to IT-based distance coordination. Proposition 1 suggests that existing routines should be given proper attention before implementation. They may not be transferable to a telemedicine context, but adjustments, such as standardization and protocols, should target the key routines impaired in a telemedicine context. Proposition 2 suggests that coordination challenges when moving to telemedicine are less about enabling proximity through elaborated communication systems than they are about managing the relational aspects of coordination, building trust between stakeholders, notably through the establishment of agreed upon standards. Finally, propositions 3 and 4 suggest that the issue of accountability for distant cases cannot be taken for granted. Healthcare managers must acknowledge the role of proximity in accountability, and the cultural and organizational barriers to moving to another model should not be underestimated.

These findings are likely transferrable to other forms of distance work, outsourcing or virtual teams. Routines, familiarity and accountability may explain the challenges to coordination in these contexts as well. Some forms of resistance to telework may stem from this pervasive perception that accountability is related to proximity.

Results of this study must be interpreted with caution considering its methodological limitations. For one thing, the complexity examined is difficult to represent simply [14]. Although we took into account all of the interviews and reported our findings in detail, we could not present all of the details and nuances from the field and had to simplify the data collected. Another limitation is that we relied mostly on the accounts of interviewees. This lends the data to biases in terms of what they recall, and in terms of their personal perspective on how they coordinate. We tried to limit these biases by triangulating our data source, using multiple respondents from each site, from multiple perspectives (consulting and referring, technologists, surgeons and pathologists, respondents in favor or reluctant to use telepathology), and by using observations. Further research could attempt to confirm these preliminary findings, providing further validity and reliability. Our findings have face validity but are also limited since they are based on a single case study [14]. We only examine one specific form of telemedicine, telepathology, and we must therefore be cautious as to the generalizability of our findings. Nevertheless, most forms of telemedicine involve coordination with professional stakeholders outside of the healthcare site. Future research could investigate other forms of telemedicine, and also other forms of telework where similar coordinating challenges may emerge.

\section{Conclusions}

This study investigates the transformation in coordination between collocated and telemedicine context. Several major transformations emerged from our case analysis. First, predictability is ensured through plans and protocols rather than through routines in a telemedicine setting. Second, the lack of familiarity in a telemedicine network needs to be offset by clear standards to ensure common understanding between stakeholders. Third, even in a telemedicine context, proximity retains a role in determining accountability, even when it is not formalized. Finally, coordination in traditional settings relies on forms of collective, crossprofessional accountability that does not translate well into a telemedicine context. Therefore, coordination in a telemedicine setting relies more on individual accountabilities and sharper boundaries between professional groups. Rehearsing, standardizing, formalizing, and building stable and trusted processes can be a substitute to support coordination in telemedicine environments. In short, in the context of telemedicine, successful or effective coordination relies on fostering a work environment where members are willing to support each other as well as on clear processes and standards.

\section{References}

[1] Barley, S.R. Technology as an occasion for structuring: Evidence from observations of CT scanners and the social order of radiology departments. ASQ 31, 1 (1986), 78-108.

[2] Barrett, M., Oborn, E., Orlikowski, W.J., and Yates, J.A. Reconfiguring boundary relations: Robotic innovations in pharmacy work. Organization Science 23, 5 (2012), 1448-1466. 
[3] Bashshur, R.L., Reardon, T.G., and Shannon, G.W. Telemedicine: a new health care delivery system. Annual Review of Public Health 21, 1 (2000), 613-637.

[4] BCC Research. Global Markets for Telemedicine Technologies. BCC Research, 2014.

[5] Brauchli, K., Oberli, H., Hurwitz, N., et al. Diagnostic telepathology: long-term experience of a single institution. Virchows Archives 444, 5 (2004), 403-409.

[6] Bruns, H. Working alone together. Coordination in collaboration across domains of expertise. Academy of Management Journal 56, 1, (2012), 62-83.

[7] Cartwright, L. Reach out and heal someone: telemedicine and the globalization of health care. Health 4, 3 (2000), 347-377.

[8] Daft, R.L., Lengel, R.H., and Trevino, L.K. Message equivocality, media selection, and manager performance: Implications for information systems. MIS Quarterly 11, 3, (1987), 355-366.

[9] Dubé, L. and Paré, G. Rigor in information systems positivist case research: Current practices, trends, and recommendations. MIS Quarterly 27, 4, (2003), 597-636.

[10] Eisenhardt, K.M. Building theories from case study research. Academy of Management Review 14, 4 (1989), 532-550.

[11] Faraj, S. and Xiao, Y. Coordination in fast-response organizations. Management Science, (2006), 1155-1169.

[12] Gherardi, S. Telemedicine: A practice-based approach to technology. Human Relations 63, 4 (2010), 501-524.

[13] Gittell, J.H. Coordinating mechanisms in care provider groups: Relational coordination as a mediator and input uncertainty as a moderator of performance effects. Management Science, (2002), 1408-1426.

[14] Hodkinson, P. and Hodkinson, H. The strengths and limitations of case study research. Learning and Skills Development Agency Conference, (2001), 5-7.

[15] Klein, K.J., Ziegert, J.C., Knight, A.P., and Xiao, Y. Dynamic delegation: Shared, hierarchical, and deindividualized leadership in extreme action teams. Administrative Science Quarterly 51, 4 (2006), 590-621.

[16] LeRouge, C. and Garfield, M.J. Crossing the telemedicine chasm: have the US barriers to widespread adoption of telemedicine been significantly reduced? International Journal of Environmental Research and Public Health 10, 12 (2013), 6472-6484.

[17] Lowry, P.B., Roberts, T.L., Dean, D.L., and Marakas, G. Toward Building Self-Sustaining Groups in PCR-based
Tasks through Implicit Coordination: The Case of Heuristic Evaluation. JAIS 10, 3 (2009), 170-195.

[18] Meyer, J. and Paré, G. Telepathology Impacts and Implementation Challenges: A Scoping Review. Archives of Pathology \& Laboratory Medicine 139, 12 (2015), 15501557.

[19] Meyer, J., Paré, G., Trudel, M.-C., and Têtu, B. Télémédecine et accessibilité aux soins de santé spécialisés en régions éloignées. Gestion 39, 3 (2014), 29-37.

[20] Nicolini, D. The work to make telemedicine work: A social and articulative view. Social Science \& Medicine 62, 11 (2006), 2754-2767.

[21] Nicolini, D. Stretching out and expanding work practices in time and space: The case of telemedicine. Human Relations 60, 6 (2007), 889-920.

[22] Nicolini, D. Practice as the Site of Knowing: Insights from the Field of Telemedicine. Organization Science 22, (2011), 602-620.

[23] Okhuysen, G.A. and Bechky, B.A. 10 Coordination in Organizations: An Integrative Perspective. The Academy of Management Annals 3, 1 (2009), 463-502.

[24] Klein, H.K. and Myers, M.D. A set of principles for conducting and evaluating interpretive field studies in information systems. MIS Quarterly 23, 1, (1999), 67-93.

[25] Pare, G., Meyer, J., Trudel, M.-C. and Tetu, B. Impacts of a Large Decentralized Telepathology Network in Canada. Telemedicine and e-Health 22, 3, (2016), 1-5.

[26] Patton, M.Q. Qualitative research and evaluation methods. Sage Publications, 2002.

[27] Têtu, B., Paré, G., Trudel, M.-C., et al. Whole-slide imaging-based telepathology in geographically dispersed Healthcare Networks. The Eastern Québec Telepathology project. Diagnostic Histopathology 20, 12 (2014), 462-469.

[28] Weinstein, R.S. The S-curve framework: predicting the future of anatomic pathology. Archives of Pathology \& Laboratory Medicine 132, 5 (2008), 739-742.

[29] Weinstein, R.S., Graham, A.R., Richter, L.C., et al. Overview of telepathology, virtual microscopy, and whole slide imaging: prospects for the future. Human Pathology 40, 8 (2009), 1057-1069.

[30] Yin, R.K. Case study research: Design and methods. Sage publications, 2014.

[31] Young, G.J., Charns, M.P., Daley, J., Forbes, M.G., Henderson, W., and Khuri, S.F. Best practices for managing surgical services: the role of coordination. Health Care Management Review 22, 4 (1996), 72-81. 